\section{Effect of Treatment on Trans- mission Dynamics of SARS Epidemic}

\author{
Afia Naheed ${ }^{1 *}$, Manmohan Singh ${ }^{2}$ and David Lucy ${ }^{2}$ \\ ${ }^{1}$ Mathematics Department, Riphah Institute of Computing and Applied \\ Sciences (RICAS), Riphah International University, Lahore, Pakistan \\ ${ }^{2}$ Department of Mathematics, Faculty of Science, Engineering and Tech- \\ nology, Swinburne University of Technology, Melbourne, Australia
}

\begin{abstract}
Infectious diseases are the focus of much attention nowadays. Spatio-temporal study of the spread of their pathogenes is proving very helpful in developing strategies to control them, especially in cases where little biological information and treatment is available. The present work is based on the numerical study of Severe Acute Respiratory Syndrome (SARS) using an open population model consisting of six compartments. Different population distributions are used to generate the numerical simulations for the disease in different situations. Stability of the system is analysed. To see the effect on disease transmission different possible cases are studied in the absence and presence of diffusion in the system. Impact of treatment on the transmission of disease is analysed.
\end{abstract}

Keywords: Epidemic modeling; Infectious disease; Partial differential equations; Reproduction number; Routh-Hurwitz criterion; Severe Acute Respiratory Syndrome (SARS); Stability analysis

\section{Introduction}

Recent years have seen an increasing trend of utilizing mathematical models for the prediction of and insight in infectious diseases. These models are considered as conceptual tools to explain the behavior of disease at different scales, and allow us to understand the spread of infection in the real world and the impact of various factors on disease dynamics. The key concepts associated with mathematical modeling, such as basic and effective reproduction number, generation time, epidemic growth rates, mortality rates, transmission rates, incubation periods, heterogeneities, disease transmission routes, risk factors for diseases spread and pre-clinical infectiousness play significant roles in the epidemiological analysis and control of diseases. The process of modeling in epidemiology has, at its heart, the same underlying philosophy and goals as ecological modeling. Both endeavors

${ }^{*}$ Corresponding author: Afia Naheed, Mathematics Department, Riphah Institute of Computing and Applied Sciences (RICAS), Riphah International University, Lahore, Pakistan, Tel: +92 03111747 424; E-mail: afia_naheed@yahoo.com

Citation: Naheed A, Singh M, Richards D (2016) Effect of Treatment on Transmission Dynamics of SARS Epidemic. J Infect Non Infect Dis 2:16.

Received: April 25, 2016; Accepted: September 22, 2016; Published: October 06, 2016 share the ultimate aim of attempting to understand the prevalence and distribution of a species, together with the factors that determine incidence, spread and persistence [1,2].

The spatio-temporal spread of infectious diseases is the most significant area of epidemic modeling. Accurate and precise mathematical models enable scientists to understand the risk factors of disease transmission and to develop workable control strategies for possible future outbreaks. There are obvious public health and/or economic benefits in understanding the infectious dynamics of diseases in humans, animals and plants. Furthermore, it is well understood how important the spatial aspect of these dynamics is to understand disease spread [3]. In the case of emerging and re-emerging outbreaks of an infectious disease, it is crucial to quantify the characteristics of a disease in order to estimate the potential threat. Accurate estimation of these characteristics relies on modified epidemiological information.

Severe Acute Respiratory Syndrome (SARS) is one of the first pandemics of the 21st century. The World Health Organization (WHO) issued a global alert for SARS on $12^{\text {th }}$ March 2003, at which point there were 150 suspected cases in 7 countries. The epidemic emerged in Guangdong Province in mainland China in November 16, 2002 and, on February 26 of the following year, new reports of SARS outbreaks came from Hong Kong and Vietnam. This contagious disease spread across the world through air travel and, as of June 13, 2003, the cumulative number of probable cases of SARS worldwide, reached 8,454 with 792 deaths [4]. Hong Kong bore a large proportion of this mortality and morbidity burden. In Hong Kong alone, 1755 probable SARS cases and 302 SARS related deaths were recorded from 15 February to $31^{\text {st }}$ May 2003. SARS was caused by a coronavirus called SARS-Cov. As SARS was not previously endemic in humans, it seemed likely that SARS-Cov was a virus of animals that had crossed the species barrier to humans in the recent past [5]. Having originated in animals the virus then became efficient at human-to-human transmission which helped to develop the disease around the globe. SARS was brought under control at the end of 2003 but in 2004 a few cases emerged, either by accidental release of the virus from laboratories or from infected animals. It is difficult to make predictions regarding the resurgence of SARS, but current information suggests that the greatest risk of the reemergence of the disease may derive from animal reservoirs [5]. Recently, in 2012, a group of international scientists has found a SARSlike virus retrieved from a Chinese horseshoe bat, giving weight to the theory that these bats are the ultimate source of the virus that killed more than 900 people around the globe in 2003 [6].

Although much effort has been devoted to find the cure for SARS, there are still no effective drugs or vaccines for the disease. In this situation mathematical modeling permits the quantitative assessment of the epidemic potential of SARS and the effectiveness of measures, used to control disease transmission. Donnelly et al. [7] assessed the epidemiology of SARS in Hong Kong. They estimated the key epidemiological parametric distributions using integrated data bases constructed from several sources. These data bases contain information about epidemiological, demographic and clinical variables and provided the baseline for the parameters of SARS 
models by Chowell et al., [8,9], Gummel et al., [10], Riley et al., [11], Yan et al., [12] and Lipstich et al. [13]. Chowell et al., [8] fitted a compartmental model for the SARS epidemic to the data from Toronto, Hong Kong and Singapore. Chowell predicted the behavior of the disease and the role of diagnosis and isolation as a control mechanism in these regions, showing the differences among the epidemic dynamics occurred in these three cities. Riley et al., [11] and Lipstich et al., [13] used dynamic models for the respective transmission dynamics of SARS in Hong Kong and Singapore. Even though these models were complex, they allowed researchers to calculate numerous epidemiologically important parameters in order to assess the potential danger of the epidemic. Wallinga and Teunis [14] developed a likelihood-based estimation procedure that infers the temporal pattern of effective reproduction numbers from an observed epidemic curve. Zhou Y et al., [4] formulated a discrete mathematical model to investigate the transmission of SARS and estimated the parameters of the model on the basis of statistical data. Numerical simulations describing the transmission process for SARS in China have been carried out. Wang $\mathrm{W}$ and Ruan S proposed a mathematical model to simulate the SARS outbreak in Beijing by estimating the reproduction number and other important epidemiological parameters using the available data [15]. Xia et al., analysed the pattern of SARS and predicted the course of the SARS epidemic by establishing a compartmental model using data from Guangdong and Hong Kong [16]. Yang et al., also used a compartmental model to describe the SARS epidemic in spatio-temporal dimensions to determine whether people travelling in buses and trains infect one another or not [17]. They concluded that SARS can spread through people travelling in buses and trains.

Any treatment of infectious disease, SARS can give rise to many important questions such as, how treatment will effect disease transmission dynamics? Will it help to control the disease? Will the intensity of the disease be same or not. In order to answer such questions, we have developed an SEIJTR model for SARS in this paper. Here we investigate the transmission of SARS in the presence of treatment. A treatment class is included in the previous SEIJR model [18]. The values of the new parameters are calculated for the SARS epidemic using the data that appeared in Hong Kong 2003 [19]. This compartmental model includes susceptible, exposed, infected, diagnosed, treated and recovered classes. Diffusion has been included in the system to examine its role in transmission of the disease. The compartmental model for SARS transmission is given in section 2. The numerical scheme to solve the model is described in section 3 . The stability of numerical model with and without diffusion is analysed in section 4 . section 5 shows numerical simulations. Further discussions and conclusions are given in section 6 .

\section{SEIJTR Epidemic Model}

\section{Equations}

This model of SARS [18] consists of the following system of nonlinear ordinary differential equations.

$$
\begin{aligned}
& \frac{\partial S}{\partial t}=\pi \wedge-\beta \frac{(I+q E+l J)}{N} S-\mu S+d_{1} \frac{\partial^{2} S}{\partial x^{2}} \\
& \frac{\partial E}{\partial t}=(1-\pi) \wedge+\beta \frac{(I+q E+l J)}{N} S-(\mu+\kappa) E+d_{2} \frac{\partial^{2} E}{\partial x^{2}} \\
& \frac{\partial I}{\partial t}=\kappa E-(\mu+\alpha+\delta) I+d_{3} \frac{\partial^{2} I}{\partial x^{2}}
\end{aligned}
$$

$$
\begin{aligned}
& \frac{\partial J}{\partial t}=\alpha I-\left(\mu+\gamma_{1}+\delta+\zeta\right) J+d_{4} \frac{\partial^{2} J}{\partial x^{2}} \\
& \frac{\partial T}{\partial t}=\zeta J-\left(\gamma_{2}+\mu+\delta(1-\theta)\right) T+d_{5} \frac{\partial^{2} T}{\partial x^{2}} \\
& \frac{\partial R}{\partial t}=\gamma_{1} J+\gamma_{2} T-\mu R+d_{6} \frac{\partial^{2} R}{\partial x^{2}}
\end{aligned}
$$

with initial conditions

$\mathrm{S}(0)=\mathrm{S}_{0}, \mathrm{E}(0)=\mathrm{E}_{0}, \mathrm{I}(0)=\mathrm{I}_{0}, \mathrm{~J}(0)=\mathrm{J}_{0}, \mathrm{~T}(0)=\mathrm{T}_{0}$ and $\mathrm{R}(0)=\mathrm{R}_{0}$ where S,E,I, J,T and R represent susceptible, exposed, infected, diagnosed, treated and recovered classes respectively and $\mathrm{N}$ denotes the total population, $\mathrm{N}=\mathrm{S}+\mathrm{E}+\mathrm{I}+\mathrm{J}+\mathrm{T}+\mathrm{R} . \mathrm{d}_{1}, \mathrm{~d}_{2}, \mathrm{~d}_{3}, \mathrm{~d}_{4}, \mathrm{~d}_{5}$ and $\mathrm{d}_{6}$ are the diffusivity constants. The flow diagram of the SEIJTR model is given in Appendix A whereas table 1 provides description and the values of the parameters.

To scale the population size in each compartment by the total population sizes by substituting $\mathrm{s}=\mathrm{S} / \mathrm{N}, \mathrm{e}=\mathrm{E} / \mathrm{N}, \mathrm{i}=\mathrm{I} / \mathrm{N}, \mathrm{j}=\mathrm{J} / \mathrm{N}$, $\mathrm{t}=\mathrm{T} / \mathrm{N}, \mathrm{r}=\mathrm{R} / \mathrm{N} \Pi=\Lambda / \mathrm{N}$ giving the system of equations (7) - (12). After simplification replacing $s$ by $S$, e by $\mathrm{E}, \mathrm{i}$ by $\mathrm{I}, \mathrm{j}$ by J, $\mathrm{t}$ by $\mathrm{T}$ and $\mathrm{r}$ by $\mathrm{R}$, the following dimensionless system of equations is obtained:

$\frac{\partial s}{\partial t}=\pi \Pi-\Pi s-\beta(I+q E+l J) S+\delta(I+J+(\theta-1) T) S+d_{1} \frac{\partial^{2} s}{\partial x^{2}}$

\begin{tabular}{|c|c|c|c|}
\hline Parameter & Description & Value & Source \\
\hline$\wedge$ & $\begin{array}{l}\text { Rate at which new recruits enter the } \\
\text { population }\end{array}$ & 0.00002 per day & [19] \\
\hline$\pi$ & $\begin{array}{l}\text { Proportion of new recruits into the } \\
\text { population that are susceptible } \\
\text { (the complementary proportion are } \\
\text { infective) }\end{array}$ & 0.85 & [19] \\
\hline$\beta$ & Transmission coefficient & 0.24 & [19] \\
\hline$\mu$ & Rate of natural mortality & 0.000035 & [10] \\
\hline I & $\begin{array}{l}\text { Relative measure of reduced risk } \\
\text { among diagnosed }\end{array}$ & 0.65 & [19] \\
\hline K & $\begin{array}{l}\text { Rate of progression from exposed to } \\
\text { the infective }\end{array}$ & 0.195 & [19] \\
\hline q & $\begin{array}{l}\text { Relative measure of infectiousness } \\
\text { for exposed individuals }\end{array}$ & 0.1 & [7] \\
\hline$\alpha$ & $\begin{array}{l}\text { Rate of progression from infective to } \\
\text { diagnosed }\end{array}$ & 0.238 & [19] \\
\hline ү1 & Natural recovery rate & 0.046 & [19] \\
\hline ү2 & Recovery due to treatment & 0.05 & [19] \\
\hline$\zeta$ & Treatment rate & 0.2 & [19] \\
\hline$\delta$ & SARS-induced mortality rate & 0.024 & [19] \\
\hline$\theta$ & $\begin{array}{l}\text { Effectiveness of drugs as a reduc- } \\
\text { tion factor in disease-induced death } \\
\text { of infectious individuals }(0 \leq \theta \leq 1)\end{array}$ & 0.25 & [19] \\
\hline
\end{tabular}

$\frac{\partial E}{\partial t}=(1-\pi) \Pi+\beta(I+q E+l J) S-(\Pi+\kappa) E+\delta(I+J+(\theta-1) T) E+d_{2} \frac{\partial^{2} E}{\partial x^{2}}$

$\frac{\partial I}{\partial t}=\kappa E-(\alpha+\delta+\Pi) I+\delta(I+J+(\theta-1) T) I+d_{3} \frac{\partial^{2} I}{\partial x^{2}}$

Table 1: Biological definition and values of parameters.

$\frac{\partial J}{\partial t}=\alpha I-\left(\Pi+\gamma_{1}+\zeta\right) J+\delta(I+J+(\theta-1) T)+d_{4} \frac{\partial^{2} J}{\partial x^{2}}$

$\left.\frac{\partial T}{\partial t}=\zeta I-\left(\Pi+\gamma_{2}\right) T+\delta(I+J+(\theta-1)+(\theta-1) T) T\right)+d_{5} \frac{\partial^{2} T}{\partial x^{2}}$ 
$\frac{\partial R}{\partial t}=\gamma_{1} J+\gamma_{2} T-\Pi R+\delta(I+J+(\theta-1) T) R+d_{6} \frac{\partial^{2} R}{\partial x^{2}}$

Where $\mathrm{S}+\mathrm{E}+\mathrm{I}+\mathrm{J}+\mathrm{T}+\mathrm{R}=1$

\section{Initial and boundary conditions}

The domain of all the calculations is considered as $[-2,2]$. Boundary and initial conditions are chosen as follows:

$\frac{\partial S(-2, t)}{\partial x}=\frac{\partial E(-2, t)}{\partial x}=\frac{\partial I(-2, t)}{\partial x}=\frac{\partial J(-2, t)}{\partial x}=\frac{\partial T(-2, t)}{\partial x}=\frac{\partial R(-2, t)}{\partial x}=0$

$\frac{\partial S(2, t)}{\partial x}=\frac{\partial E(2, t)}{\partial x}=\frac{\partial I(2, t)}{\partial x}=\frac{\partial J(2, t)}{\partial x}=\frac{\partial T(2, t)}{\partial x}=\frac{\partial R(2, t)}{\partial x}=0$

$\left(\left(\mathrm{S}_{0}, \mathrm{E}_{0}, \mathrm{I}_{0}, \mathrm{~J}_{0}, \mathrm{~T}_{0}, \mathrm{R}_{0}\right) \mid \mathrm{S}_{0}=0.98 \operatorname{Sech}(5 \mathrm{x}-1), \mathrm{E}_{0}=\mathrm{J}_{0}=\mathrm{T}_{0}=\mathrm{R}_{0}=0, \mathrm{I}_{0}=0.02\right.$ Sech (5x-1), $-2 \leq \mathrm{x} \leq 2)$

$\left(\left(\mathrm{S}_{0}, \mathrm{E}_{0}, \mathrm{I}_{0}, \mathrm{~J}_{0}, \mathrm{~T}_{0}, \mathrm{R}_{0}\right) \mid \mathrm{S}_{0}=0.97 \operatorname{Sech}(-5 \mathrm{x}-1)^{2}\right), \mathrm{E}_{0}=\mathrm{J}_{0}=\mathrm{T}_{0}=\mathrm{R}_{0}=0, \mathrm{I}_{0}=$ $\left.0.03 \exp \left(-5(\mathrm{x}+1)^{2}\right),-2 \leq \mathrm{x} \leq 2\right)$

$\left(\left(\mathrm{S}_{0}, \mathrm{E}_{0}, \mathrm{I}_{0}, \mathrm{~J}_{0}, \mathrm{~T}_{0}, \mathrm{R}_{0}\right) \mid \mathrm{S}_{0}=0.96 \operatorname{Sech}(15 \mathrm{x}), \mathrm{E}_{0}=\mathrm{J}_{0}=\mathrm{T}_{0}=\mathrm{R}_{0}=0,-2 \leq \mathrm{x} \leq 2\right.$ $\mathrm{I}_{0}=0,-2 \leq \mathrm{x}<-0.6$ and $-0.6<\mathrm{x}<2, \mathrm{I}_{0}=0.04,-0.6 \leq \mathrm{x} \leq 0.6$ (17)

Figures 1 and 2 show the initial "population distributions" for $S$ and I. A larger susceptible and a smaller infected proportion is concentrated towards the right half of the main domain in initial condition (i). In initial condition (ii), I has high concentration in the left half of the domain $[-2,2]$ and population $S$ has concentration on the right half of the domain $[-2,2]$. In the initial condition (iii) susceptible $S$ exists in high concentration around the middle of domain $[-2,2]$ with infected also around the middle but beyond the domain of $\mathrm{S}$.
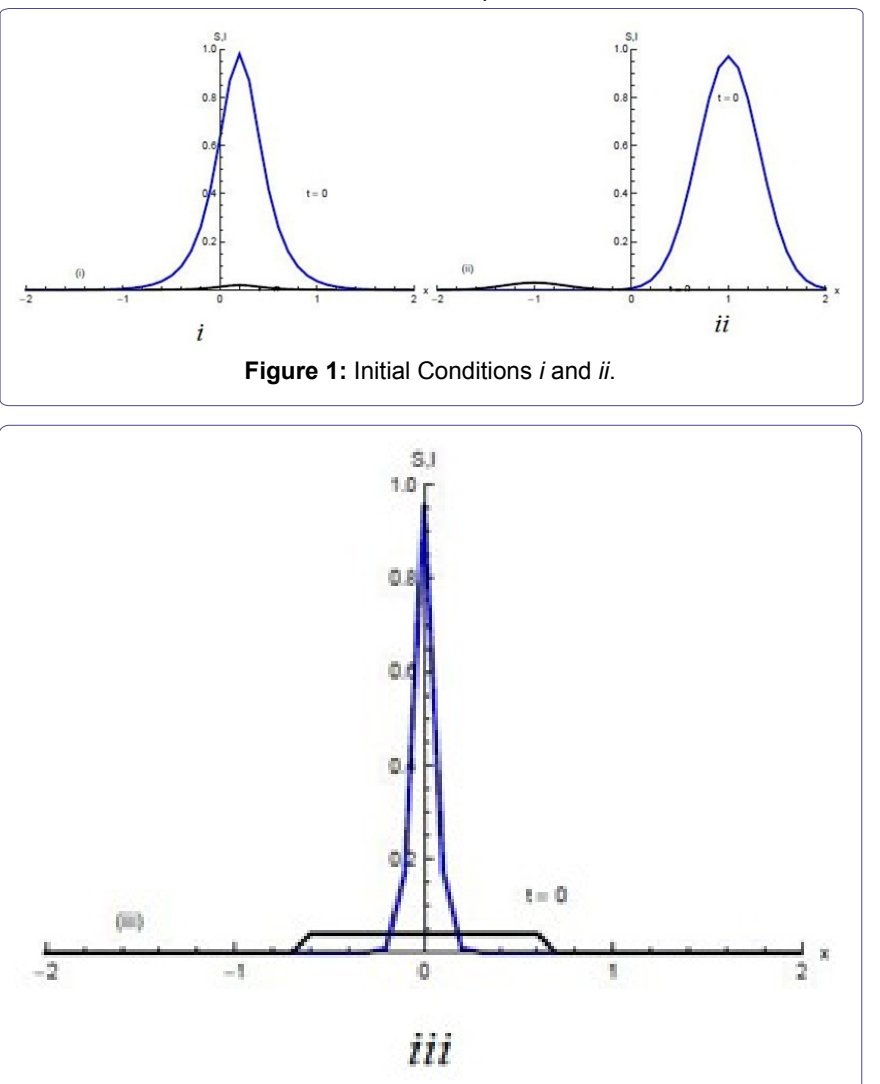

Figure 2: Initial Condition iii.

\section{Numerical Scheme}

Operator splitting method has been used to solve the SEIJTR model. According to this technique the system of equations is divided into nonlinear reaction equations and linear diffusion equations [20].
The nonlinear reaction equations to be used for the first half-time step are given as:

$$
\begin{aligned}
& \frac{1}{2} \frac{\partial S}{\partial t}=\pi \prod-\Pi S-\beta(I+q E+l J) S+\delta(I+J+(\theta-1) T) S \\
& \frac{1}{2} \frac{\partial E}{\partial t}=(1-\pi) \Pi+\beta(I+q E+l J) S-(\Pi+\kappa) E+\delta(I+J+(\theta-1) T) E \\
& \frac{1}{2} \frac{\partial I}{\partial t}=\kappa E-(\alpha+\delta+\Pi) I+\delta(I+J+(\theta-1) T) I \\
& \frac{1}{2} \frac{\partial J}{\partial t}=\alpha I-\left(\Pi+\gamma_{1}+\zeta\right) J+\delta(I+J+(\theta-1) T) J \\
& \frac{1}{2} \frac{\partial T}{\partial t}=\zeta J-\left(\Pi+\gamma_{2}\right) T+\delta(I+J+(\theta-1)+(\theta-1) T) T \\
& \frac{1}{2} \frac{\partial R}{\partial t}=\gamma_{1} J+\gamma_{2} T-\Pi R+\delta(I+J+(\theta-1) T) R
\end{aligned}
$$

The second group consists of the linear diffusion equations, to be used for the second half-time step as follows:

$$
\begin{aligned}
& \frac{1}{2} \frac{\partial S}{\partial t}=d_{1} \frac{\partial^{2} S}{\partial x^{2}} \\
& \frac{1}{2} \frac{\partial E}{\partial t}=d_{2} \frac{\partial^{2} E}{\partial x^{2}} \\
& \frac{1}{2} \frac{\partial I}{\partial t}=d_{3} \frac{\partial^{2} I}{\partial x^{2}} \\
& \frac{1}{2} \frac{\partial J}{\partial t}=d_{4} \frac{\partial^{2} J}{\partial x^{2}} \\
& \frac{1}{2} \frac{\partial T}{\partial t}=d_{5} \frac{\partial^{2} T}{\partial x^{2}} \\
& \frac{1}{2} \frac{\partial R}{\partial t}=d_{6} \frac{\partial^{2} R}{\partial x^{2}}
\end{aligned}
$$

Applying the forward Euler scheme the non-linear equations transform to

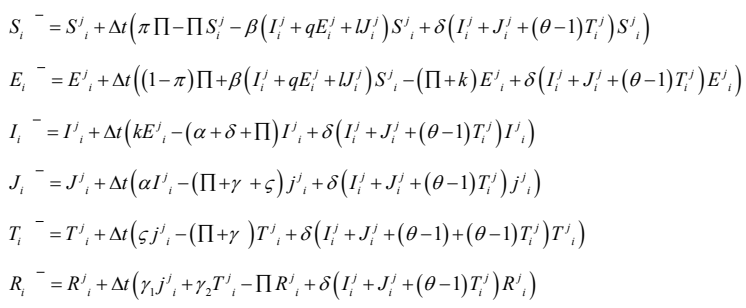

Where $S_{i}^{j}, E_{i}^{j}, I_{i}^{j}, J_{i}^{j}, T_{i}^{j}$ and $R_{i}^{j}$ are the approximated values of S, E, I, J, T and R at position $-2+i \Delta x$, for $i=0,1 \ldots \ldots$ and time $j \Delta t, j$ $=0,1, \ldots \ldots .$. and $S_{i}^{j+\frac{1}{2}}, E_{i}^{j+\frac{1}{2}}, I_{i}^{j+\frac{1}{2}}, J_{i}^{j+\frac{1}{2}}, T_{i}^{j+\frac{1}{2}}$ and $R_{i}^{j+\frac{1}{2}}$ denote their values at the first half-time step. Similarly, for the second half-time step, the linear equations transform as

$$
\begin{aligned}
& S_{i}^{j+1}=S_{i}^{j+\frac{1}{2}}+d_{1} \frac{\Delta t}{(\Delta x)^{2}}\left(S_{i-1}^{j+\frac{1}{2}}-2 S_{i}^{j+\frac{1}{2}}+S_{i+1}^{j+\frac{1}{2}}\right) \\
& E_{i}^{j+1}=E_{i}^{j+\frac{1}{2}}+d_{2} \frac{\Delta t}{(\Delta x)^{2}}\left(E_{i-1}^{j+\frac{1}{2}}-2 E_{i}^{j+\frac{1}{2}}+E_{i+1}^{j+\frac{1}{2}}\right) \\
& I_{i}^{j+1}=I_{i}^{j+\frac{1}{2}}+d_{3} \frac{\Delta t}{(\Delta x)^{2}}\left(I_{i-1}^{j+\frac{1}{2}}-2 I_{i}^{j+\frac{1}{2}}+I_{i+1}^{j+\frac{1}{2}}\right) \\
& J_{i}^{j+1}=J_{i}^{j+\frac{1}{2}}+d_{4} \frac{\Delta t}{(\Delta x)^{2}}\left(J_{i-1}^{j+\frac{1}{2}}-2 J_{i}^{j+\frac{1}{2}}+J_{i+1}^{j+\frac{1}{2}}\right) \\
& T_{i}^{j+1}=T_{i}^{j+\frac{1}{2}}+d_{5} \frac{\Delta t}{(\Delta x)^{2}}\left(T_{i-1}^{j+\frac{1}{2}}-2 T_{i}^{j+\frac{1}{2}}+T_{i+1}^{j+\frac{1}{2}}\right)
\end{aligned}
$$


$R_{i}^{j+1}=R_{i}^{j+\frac{1}{2}}+d_{6} \frac{\Delta t}{(\Delta x)^{2}}\left(R_{i-1}^{j+\frac{1}{2}}-2 R_{i}^{j+\frac{1}{2}}+R_{i+1}^{j+\frac{1}{2}}\right)$

The stability condition satisfied by the numerical method described above is given as:

$$
\frac{d_{n} \Delta t}{(\Delta x)^{2}} \leq 0.5, \quad n=1,2,3,4,5,6
$$

In each case, $\Delta x=0.1, \mathrm{~d}_{1}=0.025, \mathrm{~d}_{2}=0.01, \mathrm{~d}_{3}=0.001, \mathrm{~d}_{4}=0.0$, $\mathrm{d}_{5}=0.0, \mathrm{~d}_{6}=0.0$ and $\Delta t=0.03$ are used.

\section{Stability Analysis}

\section{Disease-Free Equilibrium (DFE)}

The basic reproduction number $\mathrm{R}_{0}$ is considered to be the threshold parameter for any DFE and is defined as "the number of secondary cases which one case would produce in a completely susceptible population". The probability of infecting a susceptible individual during one contact, duration of the infectious period, and the number of new susceptible individuals contacted per unit of time are the main factors in calculation of the reproduction number. Therefore $\mathrm{R}_{0}$ may vary remarkably for different infectious diseases and also for the same disease in different populations. The variational matrix of the system of equations (7) - (12) at the disease-free equilibrium $\mathrm{P}_{0}=(1,0,0,0,0$, $0)$, giving:

$V^{0}=\left[\begin{array}{cc}-\Pi & -q \beta \\ 0 & (q \beta-\kappa-\Pi) \\ 0 & k \\ 0 & 0 \\ 0 & 0 \\ 0 & 0\end{array}\right.$

$$
\begin{aligned}
& -\beta+\delta \\
& \beta \\
& -(\alpha+\delta+\Pi) \\
& \alpha \\
& 0 \\
& 0
\end{aligned}
$$$$
\begin{array}{cc}
-l \beta+\delta & (\theta-1) \delta \\
l \beta & 0 \\
0 & 0 \\
-\left(\gamma_{1}+\varsigma+\Pi\right) & 0 \\
\varsigma & -\left(\gamma_{2}+(\theta-1) \delta+\Pi\right) \\
\gamma_{1} & \gamma_{2}
\end{array}
$$

Trace $\left[\mathrm{V}^{0}\right]=-\alpha=+\mathrm{q} \beta-\gamma_{1}-\gamma_{2}-\delta-\zeta+\delta(\theta-1)-\kappa-6 \Pi<0$,

$\operatorname{Det}\left[\mathrm{V}^{0}\right]=\mathrm{B} \times \Pi^{2} \times \mathrm{A}$, where $\mathrm{B}=\delta(\theta-1)-\gamma_{2}-\Pi$

and $A=q \beta(\alpha+\delta+\Pi)\left(\gamma_{1}-\zeta-\Pi\right)+\beta \kappa\left(l \alpha+\gamma_{1}+\zeta+\Pi\right)-(\kappa+\Pi)(\alpha$ $+\delta+\Pi)\left(\gamma_{1}+\zeta+\Pi\right)$

As $\left.0<\theta<1 \Rightarrow \theta-1<0 \Rightarrow \delta(\theta-1)<0 \Rightarrow \delta(\theta-1)-\gamma_{2}-\Pi\right)<0$, $\Rightarrow \mathrm{B}<0$,

and for $\mathrm{R}_{0}<1, \Rightarrow \mathrm{A}<0$, Hence, Det $\left[\mathrm{V}^{0}\right]>0$.

Where $R_{0}=\frac{\beta\left(q\left(\alpha+\delta+\prod\right)\left(\gamma_{1}+\varsigma+\Pi\right)+\kappa\left(l \alpha+\gamma_{1}+\varsigma+\Pi\right)\right)}{\left(\kappa+\prod\right)\left(\alpha+\delta+\prod\right)\left(\gamma_{1}+\varsigma+\prod\right)}$

This shows that $\mathrm{P}_{0}$ is stable for $\mathrm{R}_{0}<1$. In the same way we can illustrate the stability of the endemic point for $\mathrm{R}_{0}>1$.

\section{Endemic equilibrium without diffusion}

The variational matrix of the system of equations (7) - (12) at $\mathrm{P}^{\star}$ $\left(\mathrm{S}^{\star}, \mathrm{E}^{\star}, \mathrm{I}^{\star}, \mathrm{J}^{\star}, \mathrm{T}^{\star}, \mathrm{R}^{\star}\right)$, is given by

$$
V^{*}=\left[\begin{array}{llllll}
a_{11} & a_{12} & a_{13} & a_{14} & a_{15} & a_{16} \\
a_{21} & a_{22} & a_{23} & a_{24} & a_{25} & a_{26} \\
a_{31} & a_{32} & a_{33} & a_{34} & a_{35} & a_{36} \\
a_{41} & a_{42} & a_{43} & a_{44} & a_{45} & a_{46} \\
a_{51} & a_{52} & a_{53} & a_{54} & a_{55} & a_{56} \\
a_{61} & a_{62} & a_{63} & a_{64} & a_{65} & a_{66}
\end{array}\right]
$$

a11,a12,...a66 are given in appendix B
The characteristic equation for $\mathrm{P}^{\star}\left(\mathrm{S}^{*}, \mathrm{E}^{\star}, \mathrm{I}^{*}, \mathrm{~J}^{\star}, \mathrm{T}^{\star}, \mathrm{R}^{\star}\right)$ can be written as

$$
\lambda^{6}+p_{1} \lambda^{5}+p_{2} \lambda^{4}+p_{3} \lambda^{3}+p_{4} \lambda^{2}+p_{5} \lambda+p_{6}=0
$$

where $\mathrm{p}_{1}, \mathrm{p}_{2}, \mathrm{p}_{3}, \mathrm{p}_{4}, \mathrm{p}_{5}, \mathrm{p}_{6}$ and the Routh-Hurwitz conditions are calculated on the basis of [21] and are given as:

$C_{1}: p_{1}>0$
$C_{2}: p_{6}>0$
$C_{3}: \frac{p_{1} p_{2}-p_{3}}{p_{1}}>0$

$C_{4}: \frac{p_{1} p_{2} p_{3}-p_{3}^{2}-p_{1}^{2} p_{4}-p_{1} p_{5}}{p_{1} p_{2}-p_{3}}>0$

$C_{5}: \frac{p_{3}^{2} p_{4}-p_{2} p_{3} p_{5}+p_{5}^{2}+p_{1}^{2}\left(p_{4}^{2}-p_{2} p_{6}\right)+p_{1}\left(p_{2}^{2} p_{5}-p_{2} p_{3} p_{4}-2 p_{4} p_{5}+p_{3} p_{6}\right)}{p_{3}^{2}+p_{1}^{2} p_{4}-p_{1}+\left(p_{2} p_{3}+p_{5}\right)}>0$

$C_{6}: \frac{p_{3}^{2} p_{4} p_{5}-p_{2} p_{3} p_{5}^{2}+p_{5}^{3}-p_{3}^{3} p_{6}+p_{1}^{3} p_{6}^{2}+p_{1}^{2}\left(p_{4}^{2} p_{5}-p_{3} p_{4} p_{6}-2 p_{2} p_{5} p_{6}\right)+p_{1}\left(p_{2}^{2} p_{5}^{2}+p_{2} p_{3}\left(p_{3} p_{6}-p_{4} p_{5}\right)+p_{5}\left(3 p_{3} p_{6}-2 p_{4} p_{5}\right)\right)}{p_{3}^{3} p_{4}-p_{2} p_{3} p_{5}+p_{5}^{5}+p_{1}^{2}\left(p_{4}^{2}-p_{2} p_{6}\right)+p_{1}\left(p_{2}^{2} p_{5}-p_{2} p_{3} p_{4}-2 p_{4} p_{5}+p_{3} p_{6}\right)}>0$

Here, $\mathrm{P}_{1}, \mathrm{P}_{2}, \mathrm{P}_{3}, \mathrm{P}_{4}$, and $\mathrm{P}_{5}$ are the points of equilibrium given as:

$\mathrm{P}_{1}=(0.449403,0.000058,0.000043,0.000042,0.000122,0.386452)$

$\mathrm{P}_{2}=(0.546041,0.000048,0.000035,0.000034,0.000100,0.318624)$

$\mathrm{P}_{3}=(0.402087,0.000064,0.000061,0.000044, .000131,0.419654)$

$\mathrm{P}_{4}=(0.373092,0.000066,0.000049,0.000047,0.000139,0.440013)$

$\mathrm{P}_{5}=(0.483878,0.000054,0.000033,0.000039,0.00012,0.362260)$

The numerical values of $\mathrm{C}_{1}, \mathrm{C}_{2}, \mathrm{C}_{3}, \mathrm{C}_{4}, \mathrm{C}_{5}$ and $\mathrm{C}_{6}$ on these points of equilibrium are given in table 5 in appendix $\mathrm{A}$.

\section{Endemic equilibrium with diffusion}

To calculate the small perturbations $S_{1}(x, t), E_{1}(x, t), I_{1}(x, t), J_{1}(x, t)$, $\mathrm{T}_{1}(\mathrm{x}, \mathrm{t})$ and $\mathrm{R}_{1}(\mathrm{x}, \mathrm{t})$ the equations $7-12$ are linearised about the point of equilibrium $\mathrm{P}^{\star}\left(\mathrm{S}^{\star}, \mathrm{E}^{\star}, \mathrm{I}^{\star}, \mathrm{J}^{\star}, \mathrm{T}^{\star}, \mathrm{R}^{\star}\right)$ as described in [22,23], giving

$\frac{\partial S_{1}}{\partial t}=a_{11} S_{1}+a_{12} E_{1}+a_{13} I_{1}+a_{14} J_{1}+a_{15} T_{1}+a_{16} R_{1}+d_{1} \frac{\partial^{2} S_{1}}{\partial x^{2}}$

$\frac{\partial E_{1}}{\partial t}=a_{21} S_{1}+a_{22} E_{1}+a_{23} I_{1}+a_{24} J_{1}+a_{25} T_{1}+a_{26} R_{1}+d_{2} \frac{\partial^{2} E_{1}}{\partial x^{2}}$

$\frac{\partial I_{1}}{\partial t}=a_{31} S_{1}+a_{32} E_{1}+a_{33} I_{1}+a_{34} J_{1}+a_{35} T_{1}+a_{36} R_{1}+d_{3} \frac{\partial^{2} I_{1}}{\partial x^{2}}$

$\frac{\partial J_{1}}{\partial t}=a_{41} S_{1}+a_{42} E_{1}+a_{43} I_{1}+a_{44} J_{1}+a_{45} T_{1}+a_{46} R_{1}+d_{4} \frac{\partial^{2} J_{1}}{\partial x^{2}}$

$\frac{\partial T_{1}}{\partial t}=a_{51} S_{1}+a_{52} E_{1}+a_{53} I_{1}+a_{54} J_{1}+a_{55} T_{1}+a_{56} R_{1}+d_{5} \frac{\partial^{2} T_{1}}{\partial x^{2}}$

$\frac{\partial R_{1}}{\partial t}=a_{61} S_{1}+a_{62} E_{1}+a_{63} I_{1}+a_{64} J_{1}+a_{65} T_{1}+a_{66} R_{1}+d_{6} \frac{\partial^{2} R_{1}}{\partial x^{2}}$

where $a_{11}, a_{12}, a_{13}$...etc are the elements of the variational matrix $V^{*}$ calculated using the method described in [24]. We assume the existence of a Fourier series solution of equations (44) - (49), of form:

$$
\begin{aligned}
& S_{1}(x, t)=\sum_{k} S_{k} e^{\lambda t} \cos (k x) \\
& E_{1}(x, t)=\sum_{k} E_{k} e^{\lambda t} \cos (k x) \\
& I_{1}(x, t)=\sum_{k} I_{k} e^{\lambda t} \cos (k x) \\
& J_{1}(x, t)=\sum_{k} J_{k} e^{\lambda t} \cos (k x) \\
& T_{1}(x, t)=\sum_{k} T_{k} e^{\lambda t} \cos (k x) \\
& R_{1}(x, t)=\sum_{k} R_{k} e^{\lambda t} \cos (k x)
\end{aligned}
$$


where $\mathrm{k}=\frac{n \pi}{2},(\mathrm{n}=1,2,3, \ldots \ldots)$ is the wave number for node $\mathrm{n}$. Substituting the values of $\mathrm{S}_{1}, \mathrm{E}_{1}, \mathrm{I}_{1}, \mathrm{~J}_{1}, \mathrm{~T}_{1}$ and $\mathrm{R}_{1}$ into the equations (44) -(49), the equations are transformed into

$$
\begin{aligned}
& \sum_{k}\left(a_{11}-d_{1} k^{2}-\lambda\right) S_{k}+\sum_{k} a_{12} E_{k}+\sum_{k} a_{13} I_{k}+\sum_{k} a_{14} J_{k}+\sum_{k} a_{15} T_{k}=0 \\
& \sum_{k} a_{21} S_{k}+\sum_{k}\left(a_{22}-d_{2} k^{2}-\lambda\right) E_{k}+\sum_{k} a_{23} I_{k}+\sum_{k} a_{24} J_{k} \sum_{k} a_{25} T_{k}=0 \\
& \sum_{k} a_{32} E_{k}+\sum_{k}\left(a_{33}-d_{3} k^{2}-\lambda\right) I_{k}+\sum_{k} a_{34} J_{k}+\sum_{k} a_{35} T_{k}=0 \\
& \sum_{k} a_{43} I_{k}+\sum_{k}\left(a_{44}-d_{4} k^{2}-\lambda\right) J_{k}+\sum_{k} a_{45} T_{k}=0 \\
& \sum_{k} a_{53} I_{k}+\sum_{k} a_{54} J_{k}+\sum_{k}\left(a_{55}-d_{5} k^{2}-\lambda\right) T_{k}=0 \\
& \sum_{k} a_{63} I_{k}+\sum_{k} a_{64} J_{k}+\sum_{k} a_{65} T_{k}+\sum_{k}\left(a_{66}-d_{6} k^{2}-\lambda\right) R_{k}=0
\end{aligned}
$$

The variational matrix $\mathrm{V}$ for the equations (56) - (61)

$$
V=\left[\begin{array}{cccccc}
a_{11}-d_{1} k^{2} & a_{12} & a_{13} & a_{14} & a_{15} & 0 \\
a_{21} & a_{22}-d_{2} k^{2} & a_{23} & a_{24} & a_{25} & 0 \\
0 & a_{32} & a_{33}-d_{3} k^{2} & a_{34} & a_{35} & 0 \\
0 & 0 & a_{43} & a_{44}-d_{4} k^{2} & a_{45} & 0 \\
0 & 0 & a_{53} & a_{54} & a_{55}-d_{5} k^{2} & 0 \\
0 & 0 & a_{63} & a_{64} & a_{65} & a_{66}-d_{6} k^{2}
\end{array}\right]
$$

The characteristic equation for the variational matrix $\mathrm{V}$ is given as

$$
\lambda^{6}+q_{1} \lambda^{5}+q_{2} \lambda^{4}+q_{3} \lambda_{3}+q_{4} \lambda+q_{2}+q_{5} \lambda+q_{1}+q_{6}=0
$$

where $\mathrm{q}_{1}, \mathrm{q}_{2}, \mathrm{q}_{3}, \mathrm{q}_{4}$ and $\mathrm{q}_{5}$ and $\mathrm{q}_{6}$ are calculated by the technique used in [24]. The Routh-Hurwitz Conditions are given in appendix B. The numerical values of Routh-Hurwitz criteria on points of equilibrium in the presence of diffusion are given in table 6 in appendix A.

\section{Excited mode and bifurcation value}

The technique used in [18] is used to calculate the first excited mode of the oscillation $\mathrm{n}$. According to the description of mode of excitation the curve.

$f(\beta)=A+B+(C D+E)$

where $A=q_{3}{ }^{2} q_{4} q_{5}-q_{2} q_{3} q_{5}{ }^{2}+q_{5}{ }^{3}-q_{3}{ }^{3} q_{6}+q_{1}{ }^{3} q_{6}{ }^{2}, B=q_{1}{ }^{2}\left(q_{4}{ }^{2} q_{5}-q_{3} q_{4} q_{6}\right.$ $\left.-2 \mathrm{q}_{2} \mathrm{q}_{5} \mathrm{p}_{6}\right), \mathrm{C}=\mathrm{q}_{1}\left(\mathrm{q}_{2}^{2} \mathrm{q}_{5}^{2}+\mathrm{q}_{2} \mathrm{q}_{3}\right), \mathrm{D}=-\mathrm{q}_{4} \mathrm{q}_{5}+\mathrm{q}_{3} \mathrm{q}_{6}, \mathrm{E}=\mathrm{q}_{1} \mathrm{q}_{5}\left(-2 \mathrm{q}_{4} \mathrm{q}_{5}+\right.$ $\left.3 \mathrm{q}_{3} \mathrm{q}_{6}\right) . \mathrm{n}=1$ represents the first mode of excitation as being closest to the $\beta$-axis. It is observed that the bifurcation value of transmission coefficient, $\beta$ and rate of progression from infective to diagnosed, $\alpha$ increases with diffusion as compared to the system without diffusion.

\section{Numerical Solutions}

Five cases with different values of $\beta$, the transmission coefficient and $\alpha$, rate of progression from infective to diagnosed, are summarized in table 7 given in appendix A. For all the cases given in table 7, numerical solutions are calculated for the SEIJTR model both in the absence and presence of diffusion in the system.

\section{Solutions of SEIJTR model in the absence of diffusion (Case 1)}

Figure 3, shows the numerical solution for initial condition (i) in the absence of diffusion. It can be observed that the susceptible population decreases abruptly in the first five days of disease, with the concentration of the population fluctuating near the edges of domain $[-1,1]$. At $t=10,15,20$ days, the susceptible keep on decreasing with the passage of time. In the first five days of onset of the disease, maximum population is exposed to SARS within the domain $[-0.6,1]$. After $t=5$ days, exposed population proportion shows a rapid decrease till $t=10$ days. With the passage of time, the exposed population proportion keeps on decreasing slowly, with concentration confined to the domain $[-1,1]$ at $t=20$ days. In the first five days of onset of SARS, most of the exposed get infected. There is a very large increase in the proportion of infective in the first 5 days, concentrated in the domain $[-0.6,1]$. After $t=5$ days, infective proportion decreases quickly till $\mathrm{t}=15$ days. From $\mathrm{t}=15$ days to $\mathrm{t}=20$ days, the infected population proportion decreases slowly but remains higher than the initial infected population proportion. Infective, are largely confined to the main concentration region $[-1,1]$ throughout the period of prevailing disease. As soon as the infection spreads and some of the population becomes infective, the proportion of diagnosed cases increases quite quickly from $t=0$ to $t=5$ days. This trend continues but at a slower pace, for the next five days within the domain of concentration $[-1,1]$. The diagnosed population proportion decreases quickly after $\mathrm{t}=10$ days of the disease, but there is still a substantial proportion of diagnosed at $t=20$. Once the infected are diagnosed, they start entering the treated compartment. A small increase in the population being treated can be observed in the first five days of disease. From $t$ $=5$ days to $t=10$ days, there is a sharp increase in the proportion of population in the treated class. This proportion keeps on increasing but at a low pace and reaches its maximum at $t=15$ days. After that, the treated population proportion decreases slowly with most of this population remaining confined to the domain $[-1,1]$. At $t=20$ days, a large proportion of the population is still in the treatment compartment. Recovery is slow in the first five days of spread of SARS but increases rapidly in the next five days. A sharp increase in the proportion of recovered individuals is noticeable between $t=10,15$ and 20 days. The highest recovery is observed at $t=20$ days.

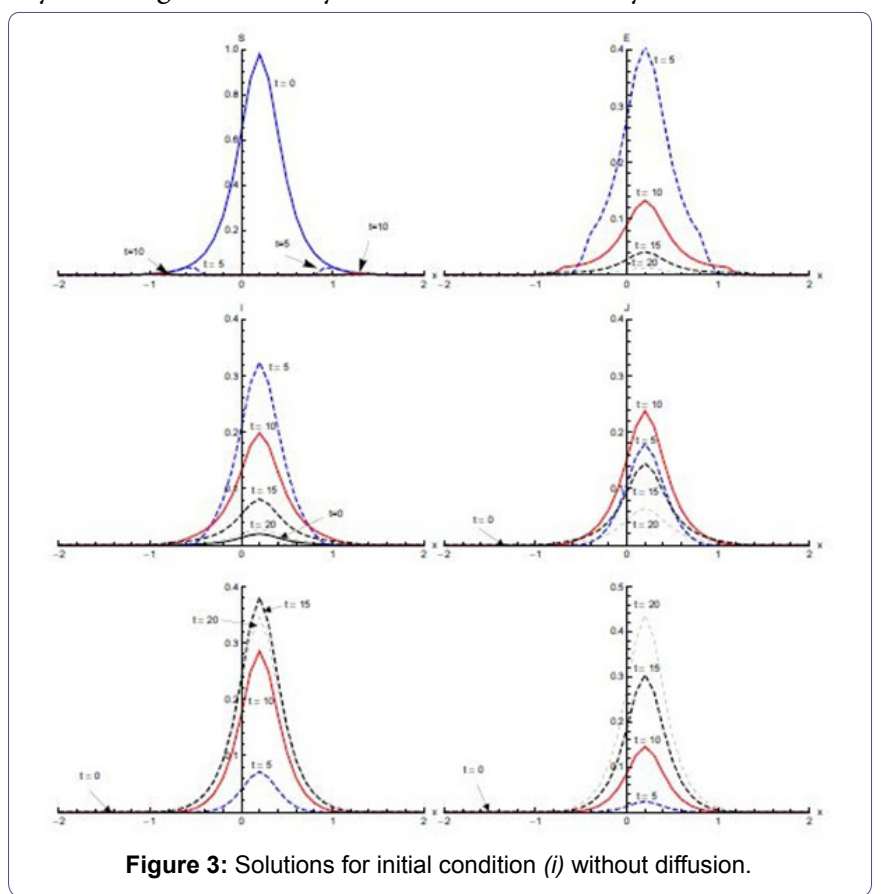

Figure 4, shows the output for initial condition (ii) in the absence of diffusion. The susceptible population, which is mainly concentrated in the domain $[0,2]$, shows sharp decline in the first five days of the spread of SARS. After five days, concentration of susceptible is confined to the edges of the domain [0,2]. A small decrease is observed from $t=5$ days onward (not visible in Figure 4). The exposed population grows in proportion remarkably fast in the first five days, showing that the rate of infection is very high during this period. Almost half of the susceptible population gets exposed to the disease 
in the first five days. Then the proportion of the population that is exposed rapidly decreases in the next five days. After $t=10$ days, a slower decrease in exposed population proportion occurs. Most of the population becomes infective in the first five days and the domain of concentration of infective moves from $[-2,0]$ to $[0,2]$. After $t=5$ days, the infective proportion starts decreasing slowly till $t=10$ days in the domain $[0,2]$. A sharp decline in infective proportion is observed in the whole domain $[-2,2]$ between $t=10$ days and $t=15$ days, which continues at slow pace thereafter. The diagnosed proportion increases over the first five days. Between $t=5$ and $t=10$ days, an increase in diagnosed population proportion is noticed in the domain $[0,2]$. In the same period, a much smaller proportion of diagnosed is also observed in the domain $[-2,0]$. A small proportion of the population is in the treatment class after five days and this proportion increases quickly in the next five days. The proportion of the population in treatment class reaches its maximum in $\mathrm{t}=15$ days and after that it decreases. There is negligible recovery in the first five days followed by a quicker recovery in the next five days in domain [-1.5,2], particularly the right half of the domain $[-2,2]$. The recovery proportion is greatest at $\mathrm{t}=20$ days.

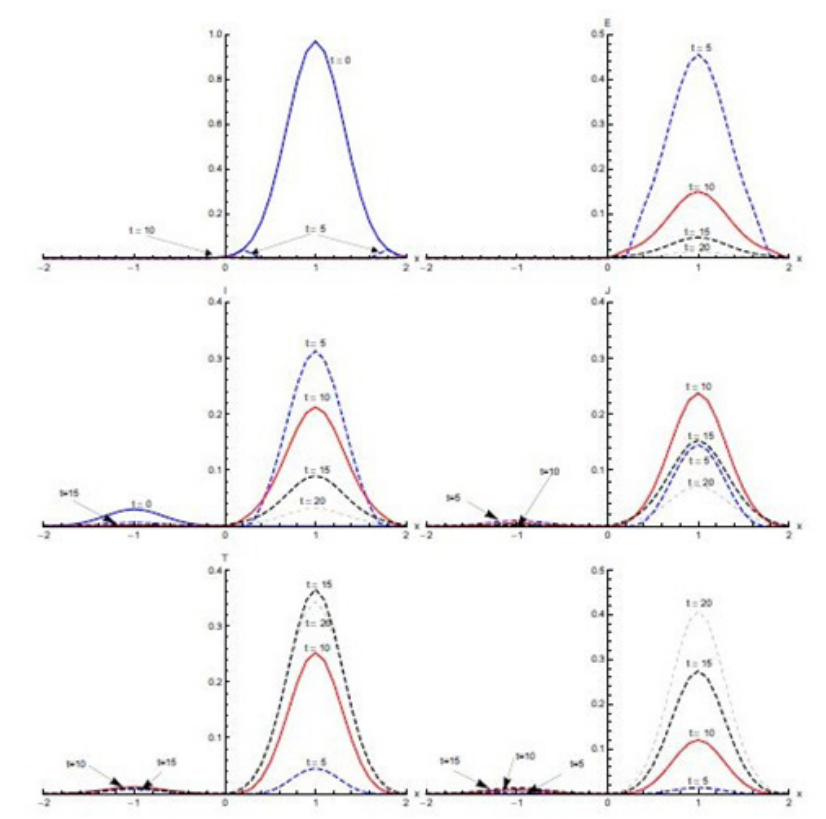

Figure 4: Solutions for initial condition (ii) without diffusion.

Figure 5 shows the results with initial condition (iii) in the absence of diffusion. In the first five days of the spread of SARS, the susceptible proportion reduces quickly to very low concentration around the edges at $x=-0.1$ and $x=0.1$. In the first five days, most of the susceptible population gets exposed to the disease. After that, there is a quick decrease in the exposed population proportion until $t=10$ days. This decreasing trend continues until $t=20$ days in the domain $[-0.1,0.1]$. The infected population proportion increases quickly at a slower pace in the domain $[-0.1,0.1]$ from its initial concentration in domain $[-0.5,0.5]$ and reaches its peak value at $\mathrm{t}=5$ days. After that, infection decreases until $t=20$ days. The diagnosed population proportion increases considerably in the first five days and is concentrated in the domain $[-0.5,0.5]$. The maximum diagnosed proportion occurs ten days after the onset of SARS. After $\mathrm{t}=10$ days, the diagnosed proportion of the population decreases. In the beginning, diagnosed individuals move slowly to the treatment class but a considerable increase in the proportion of the treated population is observed between $\mathrm{t}=5$ and $\mathrm{t}=10$ days. Recovery is extremely slow in first five days but during the next five days a substantial increase in the recovered population is observed, which continues till $\mathrm{t}=20$ days.

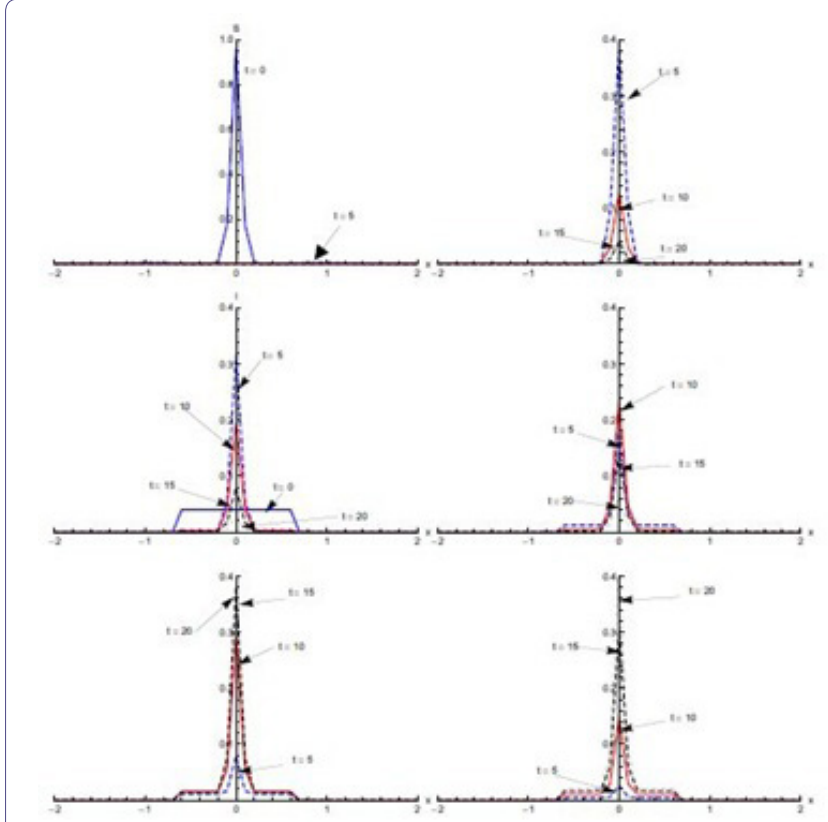

Figure 5: Solutions for initial condition (iii) without diffusion.

\section{Solutions of SEIJTR model in the presence of diffusion} (Case 1)

Figure 6, shows the output for initial condition $(i)$ in the presence of diffusion. The impact of the diffusion on the solution is well observed, as the proportion of the susceptible reduces rapidly in the first five days, with a negligible proportion of susceptible at $t=5$ days in the domain $[-2,-0.5]$ and $[0.5,2]$. Most of these susceptible in domain $[-1.5,1.5]$ are exposed to SARS during the first five days. A sharp decrease in the exposed proportion of population between $t=5$ days and $t=10$ days is observed, with the spread of the exposed population in the domain $[-2,2]$. The infected proportion of the population is maximised within $t=5$ days of spread of SARS in the domain [-1.5, 1.5]. In the next five days, infection not only decreases but also spreads rapidly in the domain $[-2,2]$. The diagnosed population proportion peaks in the first ten days of the disease and is spread across whole domain $[-2,2]$. The treatment class attains its peak value fifteen days after the spread of SARS. This is similar to the case without diffusion but with, different peak values. Due to the presence of diffusion, recovery spreads in the domain $[-2,2]$. The recovered population proportion increases rapidly after $t=5$ days and spreads to the domain $[-1.5,1.5]$. Maximum population is recovered in $\mathrm{t}=20$ days.

Figure 7, shows the solution for initial condition (ii) and with diffusion. Susceptible move from the domain $[0,2]$ to the domain $[-1.5,0]$ at $\mathrm{t}=5$ days and then to the domain $[-2,-0.5]$ at $\mathrm{t}=10$ days (not clearly visible in Figure 7). The exposed population spread in the domain $[-0.5,2]$; attaining its maximum proportion at $\mathrm{t}=5$ days and then declining sharply in the next five days, spreading in the domain $[-1,2]$. After that there is slow decrease during the next ten days. Concentration of infected population remains mostly confined to the domain $[-0.5,2]$ with a peak value at $t=5$ days. After that, the infection proportion reduces gradually. At $t=20$ days a very small proportion of the population is infected. The diagnosed population proportion also spreads in the domain $[-0.5,2]$ with its maximum occurring at $t=10$ days. A sharp increase in the treated population is observed from $t=5$ 


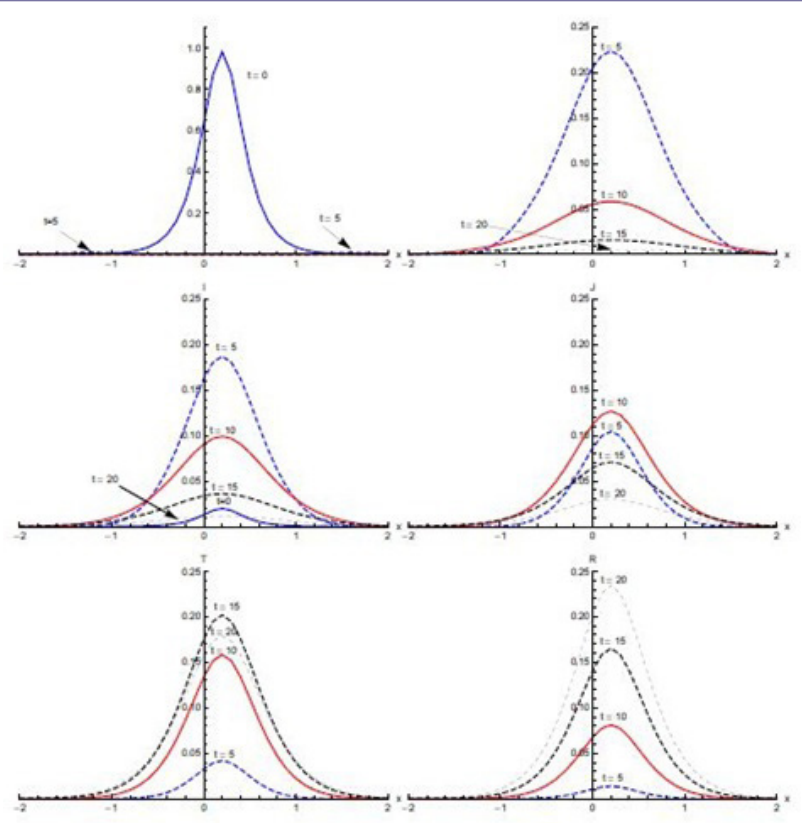

Figure 6: Solutions for initial condition (i) with diffusion.

days to $\mathrm{t}=10$ days in the domain $[-2,2]$, mostly in the domain $[-0.5,2]$. The treated proportion of the population attains its peak value fifteen days after the spread of the disease and then reduces slowly. After fifteen days recovery seems to propagate in the larger domain $[-2,2]$ but mostly in the domain $[-0.5,2]$. As compared to without diffusion case for recovered population, peak values are smaller but domain of concentration spreads.

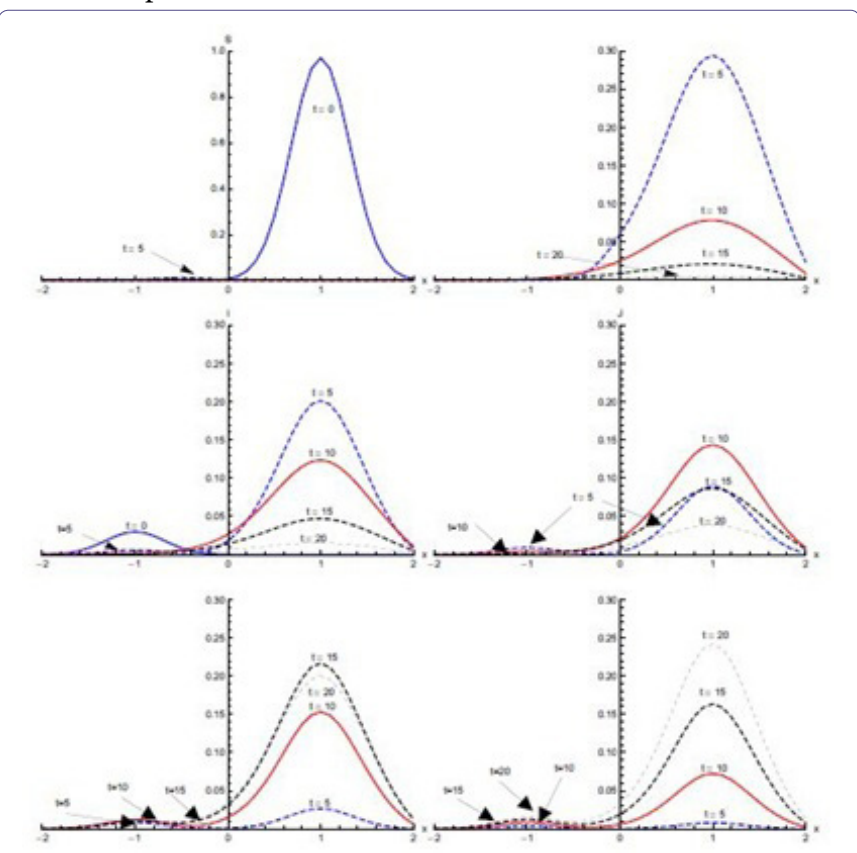

Figure 7: Solutions for initial condition (ii) with diffusion.

Figure 8 , shows the solution for initial condition (iii) with diffusion. There is a quick decline in the susceptible population proportion during the initial five days of onset of disease. Maximum population exposure to SARS occurs in the domain $[-1,1]$ in the first five days. Between $t=5$ and $t=10$ days, the peak value of the exposed population proportion decreases while the exposed spread in the domain
$[-1.5,1.5]$. Infection peaks at $t=5$ days and spreads in the domain $[-1.5,1.5]$ at $t=10$ days. Diffusion also causes diagnosed, treated and recovered population proportions to spread in the domain $[-1.5,1.5]$ with peak values at $\mathrm{t}=10,15$ and 20 days respectively.

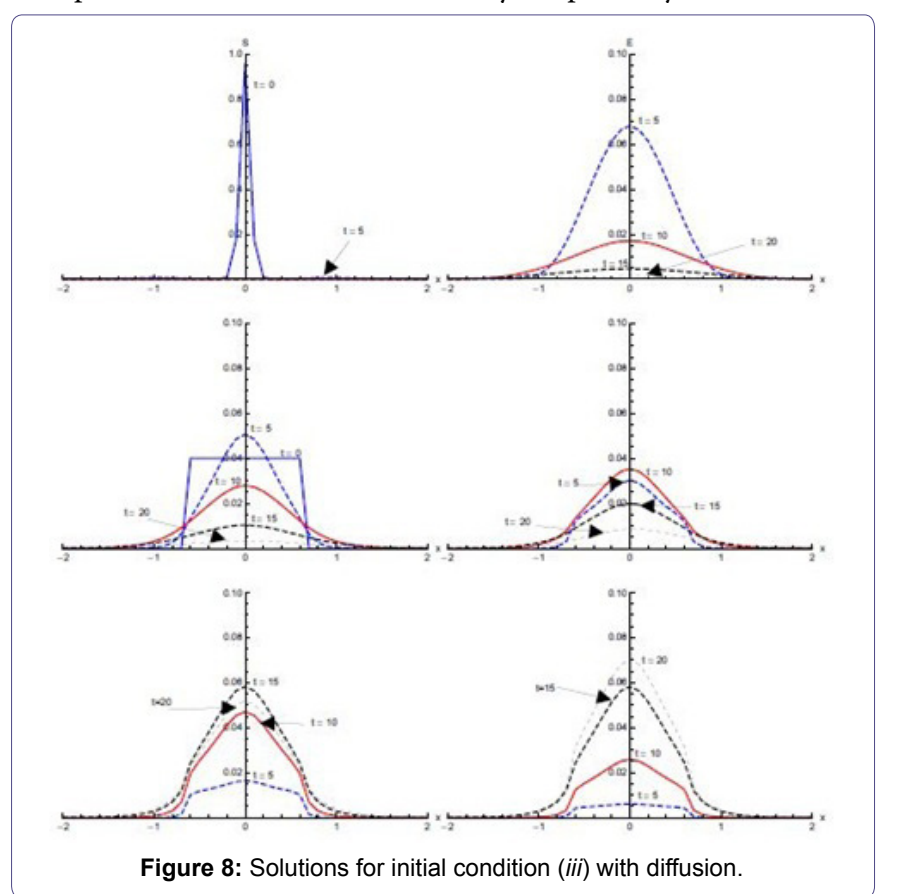

\section{Other cases}

The graphical output for Cases 2, 3, 4 and 5 is not shown because of its similarity to Case 1 . The numerical results of all cases are summarized in tables 2 and 3 . Here $S_{i}, E_{j}, I_{j}$ and $R_{j}$ for $j=(i)$, (ii) and (iii) represent peak values of the proportion of susceptible, exposed, infected and recovered population in the domain $[-2,2]$ in the absence (Table 2) and presence (Table 3 ) of diffusion for the initial population distributions (i), (ii) and (iii). The following description is based on the results given in tables 2 and 3.

Moving from Case 1 to Case 2, there is a decrease in the transmission coefficient from $\beta=0.242$ to $\beta=0.182$, as shown in table 7 (Appendix A). As a result, the susceptible population proportion show an increase in peak values for initial population distributions (i) - (iii) with and without diffusion. There is quite significant increase in the first ten days of SARS as compared to Case 1. The proportions of population exposed also show a significant increase from $t=10$ to $t=$ 15 days as compared to Case 1. Infection grows in last ten days of disease without diffusion but with diffusion infective population show an increase as compared to Case 1 from $t=10$ to $t=20$ days. There is moderate decrease in the peak value of the recovered population proportion with all initial conditions.

In Case 3, the rate of progression from infective to diagnosed, $\alpha$ is decreased from $\alpha=0.238$ to $\alpha=0.179$ as compared to Case 1 , while keeping values of $\beta$ same. There is no change in the susceptible population proportion in the absence of diffusion but, with diffusion a small increase appears at $\mathrm{t}=5$ and $\mathrm{t}=10$ days of disease as compared to Case 1. Initial conditions (i) and (iii) show a small increase in the proportion of exposed both with and without diffusion while initial condition (ii) shows a decrease at $\mathrm{t}=5$ days and great increase in next $\mathrm{t}$ $=10$ days without diffusion and small increase with diffusion. In Case 3 infected proportion values are higher than for Case 1 both with and 


\begin{tabular}{|c|c|c|c|c|c|c|c|c|c|c|c|c|c|}
\hline Case & $\mathbf{t}$ & $\mathbf{S}_{(i)}$ & $\mathbf{S}_{\text {(ii) }}$ & $\mathbf{S}_{\text {(iii) }}$ & $E_{(i)}$ & $\mathbf{E}_{(\text {ii) }}$ & $\mathbf{E}_{(\text {iii) }}$ & $I_{(i)}$ & $\mathbf{I}_{\text {(ii) }}$ & $I_{\text {(iii) }}$ & $\mathbf{R}_{(i)}$ & $\mathbf{R}_{\text {(ii) }}$ & $\mathbf{R}_{\text {(iii) }}$ \\
\hline \multirow{5}{*}{1} & 00 & 0.980 & 0.970 & 0.960 & 0.000 & 0.000 & 0.000 & 0.020 & 0.030 & 0.040 & 0.000 & 0.000 & 0.000 \\
\hline & 05 & 0.034 & 0.039 & 0.009 & 0.406 & 0.455 & 0.393 & 0.324 & 0.313 & 0.322 & 0.024 & 0.014 & 0.026 \\
\hline & 10 & 0.009 & 0.007 & 0.004 & 0.133 & 0.149 & 0.128 & 0.199 & 0.213 & 0.194 & 0.145 & 0.120 & 0.150 \\
\hline & 15 & 0.004 & 0.003 & 0.002 & 0.041 & 0.047 & 0.040 & 0.082 & 0.090 & 0.079 & 0.305 & 0.275 & 0.309 \\
\hline & 20 & 0.002 & 0.002 & 0.001 & 0.013 & 0.014 & 0.012 & 0.029 & 0.032 & 0.028 & 0.437 & 0.408 & 0.441 \\
\hline \multirow{5}{*}{2} & 00 & 0.980 & 0.970 & 0.96 & 0.000 & 0.000 & 0.000 & 0.020 & 0.030 & 0.040 & 0.000 & 0.000 & 0.000 \\
\hline & 05 & 0.053 & 0.070 & 0.009 & 0.423 & 0.479 & 0.409 & 0.324 & 0.309 & 0.322 & 0.021 & 0.012 & 0.024 \\
\hline & 10 & 0.012 & 0.014 & 0.007 & 0.138 & 0.157 & 0.133 & 0.204 & 0.219 & 0.199 & 0.139 & 0.113 & 0.145 \\
\hline & 15 & 0.005 & 0.006 & 0.002 & 0.043 & 0.049 & 0.042 & 0.085 & 0.094 & 0.082 & 0.299 & 0.267 & 0.305 \\
\hline & 20 & 0.003 & 0.002 & 0.001 & 0.013 & 0.015 & 0.013 & 0.029 & 0.034 & 0.029 & 0.433 & 0.403 & 0.437 \\
\hline \multirow{5}{*}{3} & 00 & 0.980 & 0.970 & 0.960 & 0.000 & 0.000 & 0.000 & 0.020 & 0.030 & 0.040 & 0.000 & 0.000 & 0.000 \\
\hline & 05 & 0.034 & 0.039 & 0.009 & 0.407 & 0.373 & 0.394 & 0.456 & 0.352 & 0.372 & 0.019 & 0.011 & 0.022 \\
\hline & 10 & 0.009 & 0.007 & 0.004 & 0.134 & 0.271 & 0.129 & 0.150 & 0.284 & 0.266 & 0.124 & 0.102 & 0.129 \\
\hline & 15 & 0.004 & 0.003 & 0.004 & 0.042 & 0.131 & 0.041 & 0.048 & 0.141 & 0.127 & 0.276 & 0.247 & 0.281 \\
\hline & 20 & 0.002 & 0.002 & 0.001 & 0.013 & 0.053 & 0.013 & 0.015 & 0.058 & 0.052 & 0.411 & 0.382 & 0.415 \\
\hline \multirow{5}{*}{4} & 00 & 0.980 & 0.970 & 0.960 & 0.000 & 0.000 & 0.000 & 0.020 & 0.030 & 0.040 & 0.000 & 0.000 & 0.000 \\
\hline & 05 & 0.008 & 0.037 & 0.008 & 0.217 & 0.439 & 0.383 & 0.191 & 0.315 & 0.322 & 0.025 & 0.016 & 0.028 \\
\hline & 10 & 0.007 & 0.006 & 0.002 & 0.129 & 0.144 & 0.125 & 0.195 & 0.208 & 0.191 & 0.149 & 0.125 & 0.154 \\
\hline & 15 & 0.003 & 0.002 & 0.001 & 0.040 & 0.045 & 0.039 & 0.079 & 0.087 & 0.077 & 0.308 & 0.279 & 0.313 \\
\hline & 20 & 0.002 & 0.001 & 0.001 & 0.012 & 0.014 & 0.012 & 0.028 & 0.031 & 0.027 & 0.439 & 0.412 & 0.443 \\
\hline \multirow{5}{*}{5} & 00 & 0.980 & 0.970 & 0.960 & 0.000 & 0.000 & 0.000 & 0.020 & 0.030 & 0.040 & 0.000 & 0.000 & 0.000 \\
\hline & 05 & 0.035 & 0.039 & 0.009 & 0.406 & 0.456 & 0.393 & 0.284 & 0.279 & 0.281 & 0.028 & 0.016 & 0.031 \\
\hline & 10 & 0.009 & 0.007 & 0.004 & 0.132 & 0.148 & 0.127 & 0.152 & 0.147 & 0.165 & 0.161 & 0.134 & 0.166 \\
\hline & 15 & 0.004 & 0.003 & 0.002 & 0.041 & 0.046 & 0.039 & 0.056 & 0.054 & 0.061 & 0.323 & 0.293 & 0.328 \\
\hline & 20 & 0.002 & 0.002 & 0.001 & 0.012 & 0.014 & 0.012 & 0.018 & 0.017 & 0.020 & 0.452 & 0.424 & 0.456 \\
\hline
\end{tabular}

Table 2: Peak values of Susceptible(S), Exposed(E), Infective(I) and Recovered(R) (Without Diffusion).

without diffusion. Peak values of the recovered population proportion are lower for Case 3 than Case 1.

In Case 4 , there is an increase in the transmission coefficient, $\beta$, from $\beta=0.242$ to $\beta=0.303$ as compared to Case 1 . As a result, peak susceptible proportion values in Case 4 decrease in comparison to Case 1 for initial population distributions (i) - (iii), with and without diffusion. There is also a decrease in exposed population proportion as a result of the reduction in the proportion of susceptible under all initial conditions. In particular in the absence of diffusion it occurs in the first five days of SARS, where population distribution with initial condition $(i)$ shows significant decrease in susceptible population as compared to initial conditions (ii) and (iii) while in the presence of diffusion this decrease can be observed on all days. A decrease in proportion of infected population has been observed in the emergence of SARS as compared to Case 1, in the absence of diffusion. This decrease is quite significant in case of initial condition $(i)$. With diffusion in the system, the peak values of infected proportion are higher for Case 4 than Case 1 under all initial conditions. Only very small increase in recovered proportions from Case 1 to Case 4 has been observed under all three conditions (i), (ii) and (iii), without diffusion. With diffusion in the system, initial conditions ( $i$ ) and (ii) reflect a significant increase in recovery from Case 1 to Case 4, while initial condition (iii) shows a slight increase. In comparison to Case 2, it has been observed that there are lower proportions of susceptible and exposed in Case 4 . Also in comparison to Case 2, proportion of infective is slightly less with diffusion as compared to without diffusion in the system during the first five days of disease. As compared to Case 2, there is an increase in the recovered population both with and without diffusion in the system.

In Case 5, as compared to Case 1 the rate of progression from infective to diagnosed, $\alpha$ is increased from $\alpha=0.238$ to $\alpha=0.298$. In the absence of diffusion this has not affected susceptible and exposed proportion values. With the inclusion of diffusion in the system, a small increase is observed in the susceptible and exposed population proportion in the early stage of disease. A significant reduction in the proportion of infected individuals has been noticed both with and without diffusion, showing that if infection is diagnosed earlier, the population move to diagnosed compartment quickly for treatment. A large increase in the recovered population proportion is observed both with and without diffusion as compared to Case 1. In comparison to Case 3, lower proportion of exposed and infective is observed in Case 5. Proportion of the population recovered both with and without diffusion, has been observed to be higher in Case 5 as compared to Case 3.

\section{Discussion and Conclusion}

The SEIJTR model for numerical study of the SARS [18] epidemics is used with diffusion and treatment included in the system to explore the effects of their availability on the spread of disease (Figure 9). Three different initial conditions have been used to examine the effects on transmission of the disease under different population distributions. Operator splitting technique is used to calculate the numerical solutions of the differential equations. The Routh-Hurwitz 


\begin{tabular}{|c|c|c|c|c|c|c|c|c|c|c|c|c|c|}
\hline Case & $\mathbf{t}$ & $\mathbf{S}_{(\mathrm{i})}$ & $S_{(i i)}$ & $\mathbf{S}_{\text {(iii) }}$ & $E_{(i)}$ & $E_{(i i)}$ & $\mathbf{E}_{(\text {iii) }}$ & $I_{(i)}$ & $\mathbf{I}_{\text {(ii) }}$ & $I_{\text {(iii) }}$ & $\mathbf{R}_{(\mathbf{i})}$ & $\mathbf{R}_{\text {(ii) }}$ & $\mathbf{R}_{\text {(iii) }}$ \\
\hline \multirow{5}{*}{1} & 00 & 0.980 & 0.970 & 0.960 & 0.000 & 0.000 & 0.000 & 0.020 & 0.030 & 0.040 & 0.000 & 0.000 & 0.000 \\
\hline & 05 & 0.00986 & 0.01191 & 0.00706 & 0.22313 & 0.29394 & 0.06802 & 0.18704 & 0.20155 & 0.05063 & 0.01379 & 0.00794 & 0.00613 \\
\hline & 10 & 0.00108 & 0.00173 & 0.00129 & 0.05818 & 0.07859 & 0.01705 & 0.09902 & 0.12357 & 0.02796 & 0.08119 & 0.07235 & 0.02591 \\
\hline & 15 & 0.00013 & 0.00067 & 0.00035 & 0.01584 & 0.02141 & 0.00467 & 0.03612 & 0.04704 & 0.01034 & 0.16517 & 0.16339 & 0.04998 \\
\hline & 20 & 0.00008 & 0.00032 & 0.00017 & 0.00445 & 0.00593 & 0.00138 & 0.01168 & 0.01541 & 0.00343 & 0.23444 & 0.24169 & 0.07034 \\
\hline \multirow{5}{*}{2} & 00 & 0.980 & 0.970 & 0.960 & 0.000 & 0.000 & 0.000 & 0.020 & 0.030 & 0.040 & 0.000 & 0.000 & 0.000 \\
\hline & 05 & 0.01416 & 0.01892 & 0.01175 & 0.23344 & 0.31042 & 0.07408 & 0.18182 & 0.19248 & 0.04489 & 0.01161 & 0.0059 & 0.00552 \\
\hline & 10 & 0.00171 & 0.00252 & 0.00201 & 0.06135 & 0.0835 & 0.01909 & 0.10112 & 0.12661 & 0.02878 & 0.07472 & 0.06503 & 0.02318 \\
\hline & 15 & 0.00021 & 0.00096 & 0.00054 & 0.01679 & 0.02284 & 0.0053 & 0.03754 & 0.04916 & 0.01113 & 0.15645 & 0.15331 & 0.04589 \\
\hline & 20 & 0.00009 & 0.00044 & 0.00023 & 0.00473 & 0.00635 & 0.00157 & 0.01226 & 0.01626 & 0.00378 & 0.22495 & 0.23083 & 0.06566 \\
\hline \multirow{5}{*}{3} & 00 & 0.980 & 0.970 & 0.960 & 0.000 & 0.000 & 0.000 & 0.020 & 0.030 & 0.040 & 0.000 & 0.000 & 0.000 \\
\hline & 05 & 0.00996 & 0.01204 & 0.00712 & 0.22348 & 0.29444 & 0.06814 & 0.21454 & 0.22479 & 0.05868 & 0.01100 & 0.00621 & 0.00499 \\
\hline & 10 & 0.00109 & 0.00174 & 0.0013 & 0.05851 & 0.07904 & 0.01711 & 0.13599 & 0.16516 & 0.03819 & 0.06908 & 0.06095 & 0.02217 \\
\hline & 15 & 0.00013 & 0.00068 & 0.00035 & 0.016 & 0.02165 & 0.00469 & 0.05891 & 0.07484 & 0.01665 & 0.14766 & 0.14526 & 0.04453 \\
\hline & 20 & 0.00008 & 0.00032 & 0.00017 & 0.00451 & 0.00602 & 0.00138 & 0.02229 & 0.02882 & 0.00641 & 0.21674 & 0.22296 & 0.06451 \\
\hline \multirow{5}{*}{4} & 00 & 0.980 & 0.970 & 0.960 & 0.000 & 0.000 & 0.000 & 0.020 & 0.030 & 0.040 & 0.000 & 0.000 & 0.000 \\
\hline & 05 & 0.00759 & 0.00839 & 0.0049 & 0.21664 & 0.28347 & 0.06427 & 0.19018 & 0.20684 & 0.05379 & 0.01543 & 0.00955 & 0.00669 \\
\hline & 10 & 0.00075 & 0.00132 & 0.00092 & 0.05618 & 0.07547 & 0.01587 & 0.09765 & 0.12152 & 0.02743 & 0.08576 & 0.07762 & 0.02799 \\
\hline & 15 & 0.00009 & 0.00052 & 0.00025 & 0.01523 & 0.02049 & 0.00431 & 0.03522 & 0.04567 & 0.00987 & 0.17129 & 0.17053 & 0.05301 \\
\hline & 20 & 0.00007 & 0.00025 & 0.00014 & 0.00427 & 0.00567 & 0.00127 & 0.01132 & 0.01487 & 0.00323 & 0.2411 & 0.24937 & 0.07383 \\
\hline \multirow{5}{*}{5} & 00 & 0.980 & 0.970 & 0.960 & 0.0000 & 0.000 & 0.000 & 0.020 & 0.030 & 0.040 & 0.000 & 0.000 & 0.000 \\
\hline & 05 & 0.00997 & 0.01206 & 0.00719 & 0.22333 & 0.29429 & 0.06821 & 0.16394 & 0.18103 & 0.043998 & 0.01612 & 0.00938 & 0.00704 \\
\hline & 10 & 0.00109 & 0.00175 & 0.00131 & 0.05807 & 0.07846 & 0.01709 & 0.07468 & 0.09523 & 0.02127 & 0.09009 & 0.08086 & 0.02859 \\
\hline & 15 & 0.00013 & 0.00068 & 0.00035 & 0.01576 & 0.02129 & 0.00468 & 0.02411 & 0.03193 & 0.00701 & 0.17671 & 0.17542 & 0.05352 \\
\hline & 20 & 0.00008 & 0.00032 & 0.00017 & 0.00442 & 0.00589 & 0.00138 & 0.00712 & 0.00949 & 0.00213 & 0.24523 & 0.2531 & 0.07389 \\
\hline
\end{tabular}

Table 3: Peak values of Susceptible(S), Exposed(E), Infective(I) and Recovered(R) (With Diffusion).

criterion is used to check stability of points of equilibrium. The models under investigation have two possible equilibria, namely the disease-free equilibrium and endemic equilibrium. Reproduction numbers $R_{0}$ for the various cases considered $\left(R_{0}>1\right)$ are given in table 4 . A study of bifurcation values of the transmission coefficient, $\beta$ and rate of progression from infective to diagnosed, $\alpha$ as shown in table 7 (Appendix A), indicates that the system remain stable with higher values of $\beta$ and $\alpha$ with diffusion in comparison to the system without diffusion.

\begin{tabular}{|c|c|c|c|}
\hline Case & $\boldsymbol{\beta}$ & $\boldsymbol{\alpha}$ & Value of $\mathbf{R}_{\mathbf{0}}$ \\
\hline 1 & 0.242 & 0.238 & 1.6148 \\
\hline 2 & 0.182 & 0.238 & 1.2212 \\
\hline 3 & 0.242 & 0.179 & 1.8668 \\
\hline 4 & 0.303 & 0.238 & 2.0354 \\
\hline 5 & 0.242 & 0.298 & 1.4561 \\
\hline
\end{tabular}

Table 4: Basic Reproduction Number $\mathrm{R}_{0}$.

It is observed from the values given in table 4 that transmission coefficient, $\beta$ and rate of progression from infective to diagnosed, $\alpha$ are quite sensitive parameters having significant impact on the basic reproductive number $R_{0}$. If the transmission coefficient, $\beta$ is decreased as in Case 2, the reproduction number $\mathrm{R}_{0}$ decreases significantly even though it is still greater than 1 . This causes slow transmission of infection and thus higher peak values in the infected compartment as shown in tables 2 and 3 from $t=10$ to $t=20$ days. A decrease in infective to diagnosed coefficient $\alpha$ as in Case 3 , causes a significant increase in the value of the basic reproduction number $\mathrm{R}_{0}$. Here a significant increase in the peak value of the infected proportion can be seen at $t=20$ days. An increased value of transmission coefficient, $\beta$ as in Case 4 , gives the maximum value of the reproduction number $\mathrm{R}_{0}$. Here, transmission of the infection becomes fastest in all initial conditions, as shown in the rate of decrease of values at various time steps shown in tables 2 and 3 . There are also slightly lower peak values of infected population proportion at $\mathrm{t}=20$ days, as compared to the original situation depicted in Case 1. An increase in the rate of progression from infective to diagnosed, $\alpha$ has given in Case 5 causes a slight decrease in the value of the basic reproduction number.

In initial condition ( $i$ ), without diffusion, infectives are concentrated in the domain $[-1,1]$. With and without diffusion in the system, infection spreads quickly in the first five days of onset of disease. With diffusion in the system, infected population spreads to the edges of the domain $[-2,2]$ in the first ten days, where initially there were no infectives. But a decrease in the peak values of the infected proportion occurs, showing that diffusion causes a decrease in the intensity of disease. Maximum population of diagnosed then enters treatment class at the day fifteen of the disease. In initial condition (ii), susceptible and infected proportions are in different domains initially. Here, infected not only increase with the passage of time but also move from domain $[-2,0]$ to domain $[0,2]$ without diffusion. With diffusion in the system, the infected spread to almost in the whole domain $[-2,2]$. Under initial condition (iii), there is a significant shift as the infected population move to the domain $[-0.1,0.1]$ significantly from the initial domain $[-0.6,0.6]$. Diffusion again causes the infection to spread, in the domain $[-1.5,1.5]$ but with reduced peak values. 
- The spread of the disease is affected by variation in initial the population distribution.

- Transmission coefficient, $\beta$ and rate of progression from infective to diagnosed, $\alpha$ play a crucial role in increasing or decreasing the basic reproductive number $\mathrm{R}_{0}$, thus affecting the degree of spread of disease.

- Diffusion reduces the peak values of the population in all compartments, thus reducing intensity of disease.

- Introduction of treatment immensely affects the transmission, making it faster and, in the process increasing recovery significantly [23].

\section{Acknowledgment}

One of the authors, Afia Naheed thanks Swinburne University of Technology for the Postgraduate Research Award.

\section{References}

1. Anderson RM, May RM (1979) Population biology of infectious diseases: Part I. Nature 280: 361-367.

2. Earn DJ, Rohani P, Grenfell BT (1998) Persistence, chaos and synchrony in ecology and epidemiology. Proc Biol Sci 265: 7-10.

3. Lawson AB (2001) Statistical Methods in Spatial Epidemiology. Int J Epidemiol 6: 1504-1505

4. Zhou Y, MA Z, Brauer F (2004) A Discrete Epidemic Model for SARS Transmission and Control in China. Math and Comput Model 40: 1491-1506.

5. Peiris JS, Yuen KY, Osterhaus AD, Stöhr K (2003) The severe acute respiratory syndrome. N Engl J Med 349: 2431-2441.

6. http://www.canada.com/health/SARSlike+viruses+found+Chinese+bats+closest+hits+2003+outbreak+virus/9102750/story.html

7. Donnelly CA, Ghani AC, Leung GM, Hedley AJ, Fraser C, et al. (2003) Epidemiological determinants of spread of causal agent of severe acute respiratory syndrome in Hong Kong. Lancet 361: 1761-1766.

8. Chowell G, Fenimore PW, Castillo-Garsow MA, Castillo-Chavez C (2003) SARS outbreaks in Ontario, Hong Kong and Singapore: the role of diagnosis and isolation as a control mechanism. J Theor Biol 224: 1-8.

9. Chowell G, Castillo-Chavez C, Fenimore PW, Kribs-Zaleta CM, Arriola L, et al. (2004) Model parameters and outbreak control for SARS. Emerg Infect Dis 10: $1258-1263$.

10. Gumel AB, Ruan S, Day T, Watmough J, Brauer F, et al. (2004) Modelling strategies for controlling SARS outbreaks. Proc Biol Sci 271: 2223-2232.

11. Riley S, Fraser C, Donnelly CA, Ghani AC, Abu-Raddad LJ, et al. (2003) Transmission dynamics of the etiological agent of SARS in Hong Kong: impact of public health interventions. Science 300: 1961-1966.

12. Yan X, Zou Y, Li J (2007) Optimal quarantine and isolation strategies in epidemics control. World Journal of Modelling and Simulation 3: 202-211.

13. Lipsitch M, Cohen T, Cooper B, Robins JM, Ma S, et al. (2003) Transmission dynamics and control of severe acute respiratory syndrome. Science 300 : 1966-1970.

14. Wallinga J, Teunis P (2004) Different epidemic curves for severe acute respiratory syndrome reveal similar impacts of control measures. Am J Epidemio 160: 509-516.

15. Wang W, Ruan S (2004) Simulating the SARS outbreak in Beijing with limited data. J Theor Biol 227: 369-379.
16. Xia JL, Yao C, Zhang GK (2003) Analysis of piecewise compartmental modeling for epidemic of SARS in Guangdon. Chinese Journal of Health Statistics 20: 162-163.

17. Yang H, Li XW, Shi H, Zhao KG, Han LJ (2003) Fly dots spreading model of SARS along transportation. J Remote Sens 7: 251-255.

18. Naheed A, Singh M, Lucy D (2014) Numerical study of SARS epidemic model with the inclusion of diffusion in the system. Applied Mathematics and Computation. 229: 480-498.

19. Naheed A, Singh M, Lucy D (2014) Parameter estimation with uncertainty and sensitivity analysis for the SARS outbreak in Hong Kong.

20. Yanenko NN (1971) The Method of Fractional Steps: The Solution of Problems of Mathematical Physics in Several Variables. Springer Berlin Heidelberg, Germany.

21. Meinsma G (1995) Elementary proof of the Routh-Hurwitz test. Systems \& Control Letters 25: 237-242.

22. Chakraborty A, Singh M, Lucy D, Ridland P (2007) Predator-prey model with prey-taxis and diffusion. Mathematical and Computer Modelling 46: 482-498.

23. Sapoukhina N, Tyutyunov Y, Arditi R (2003) The role of prey taxis in biological control: a spatial theoretical model. Am Nat 162: 61-76.

24. Samsuzzoha Md, Singh M, Lucy D (2010) Numerical study of an influenza epidemic model with diffusion. Applied Mathematics and Computation 217 $3461-3479$

\section{Appendix A:}

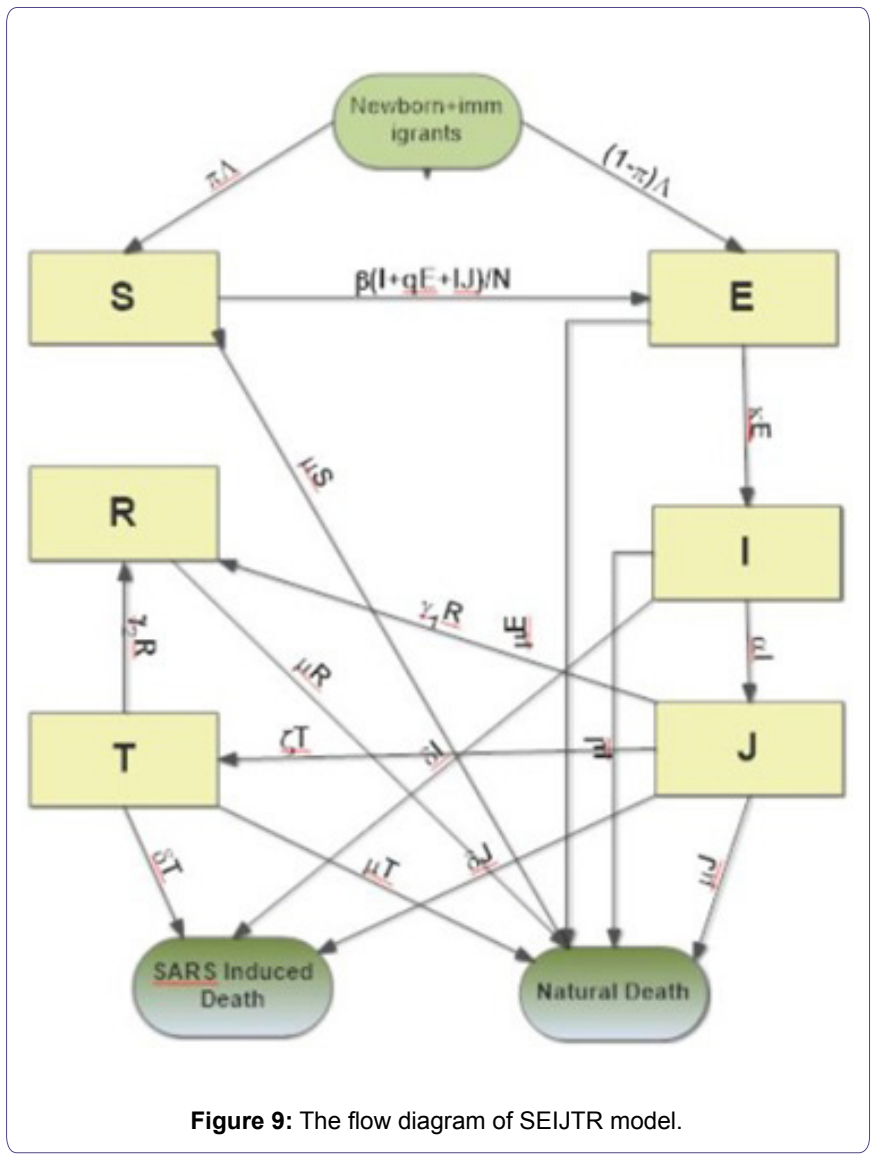




\begin{tabular}{|c|c|c|c|c|c|c|c|c|}
\hline Case & Equilibrium Point & $\mathrm{C}_{1}$ & $\mathrm{C}_{2}$ & $\mathrm{C}_{3}$ & $\mathrm{C}_{4}$ & $\mathrm{C}_{5}$ & $\mathrm{C}_{6}$ & Stable/Unstable \\
\hline 1 & $\mathrm{P}_{1}$ & 0.76036 & $4.27 \times 10^{-13}$ & 0.16736 & 0.01172 & 0.00024 & $2.54 \times 10^{-8}$ & Stable \\
\hline 2 & $\mathrm{P}_{2}$ & 0.76123 & $3.26 \times 10^{-13}$ & 0.12823 & 0.01237 & 0.00029 & $2.16 \times 10^{-8}$ & Stable \\
\hline 3 & $\mathrm{P}_{3}$ & 0.70199 & $3.79 \times 10^{-13}$ & 0.10024 & 0.00937 & 0.00017 & $2.19 \times 10^{-8}$ & Stable \\
\hline 4 & $\mathrm{P}_{4}$ & 0.75987 & $5.57 \times 10^{-13}$ & 0.126709 & 0.01133 & 0.00021 & $3.10 \times 10^{-8}$ & Stable \\
\hline 5 & $\mathrm{P}_{5}$ & 0.81903 & $4.77 \times 10^{-13}$ & 0.15788 & 0.01417 & 0.00031 & $2.93 \times 10^{-8}$ & Stable \\
\hline
\end{tabular}

Table 5: Routh-Hurwitz criteria of equilibrium without diffusion.

\begin{tabular}{|c|c|c|c|c|c|c|c|c|}
\hline Case & Equilibrium Point & $\mathbf{C}_{1}$ & $\mathbf{C}_{2}$ & $\mathbf{C}_{3}$ & $\mathbf{C}_{4}$ & $\mathbf{C}_{5}$ & $\mathbf{C}_{6}$ & $\mathbf{S t a b l e}_{\mathbf{2}}$ Un-stable \\
\hline 1 & $\mathrm{P}_{1}$ & 0.84918 & $4.51 \times 10^{-10}$ & 0.21558 & 0.02271 & 0.00107 & $2.17 \times 10^{-5}$ & Stable \\
\hline 2 & $\mathrm{P}_{2}$ & 0.85005 & $5.15 \times 10^{-10}$ & 0.21667 & 0.02335 & 0.00115 & $2.48 \times 10^{-5}$ & Stable \\
\hline 3 & $\mathrm{P}_{3}$ & 0.79082 & $3.25 \times 10^{-10}$ & 0.18731 & 0.01873 & 0.00084 & $1.59 \times 10^{-5}$ & Stable \\
\hline 4 & $\mathrm{P}_{4}$ & 0.84869 & $4.15 \times 10^{-10}$ & 0.21495 & 0.02234 & 0.00103 & $1.99 \times 10^{-5}$ & Stable \\
\hline 5 & $\mathrm{P}_{5}$ & 0.90786 & $5.85 \times 10^{-10}$ & 0.24482 & 0.02688 & 0.00131 & $2.79 \times 10^{-5}$ & Stable \\
\hline
\end{tabular}

Table 6: Routh-Hurwitz criteria of equilibrium with diffusion.

\begin{tabular}{|c|c|c|c|c|c|c|}
\hline \multirow[t]{3}{*}{ Case } & \multicolumn{2}{|c|}{ Values Considered } & \multicolumn{4}{|c|}{ Bifurcation Values } \\
\hline & \multirow[b]{2}{*}{$\beta$} & \multirow[b]{2}{*}{$\alpha$} & \multicolumn{2}{|c|}{ Without Diffusion } & \multicolumn{2}{|c|}{ With Diffusion } \\
\hline & & & $\beta$ & $\alpha$ & $\beta$ & $\alpha$ \\
\hline 1 & 0.242 & 0.238 & 0.330 & 0.071 & 0.376 & 0.088 \\
\hline 2 & 0.182 & 0.238 & 0.272 & 0.050 & 0.309 & 0.087 \\
\hline 3 & 0.242 & 0.179 & 0.320 & 0.051 & 0.365 & 0.083 \\
\hline 4 & 0.303 & 0.238 & 0.399 & 0.062 & 0.453 & 0.110 \\
\hline 5 & 0.242 & 0.298 & 0.341 & 0.087 & 0.387 & 0.115 \\
\hline
\end{tabular}

Table 7: Bifurcation Values of $\alpha$ and $\beta$.

\section{Appendix B}

\section{Endemic Equilibrium without Diffusion:}

$a_{11}=-\beta(I+q E+l J)+\delta(I+J+(\theta-1) T)-\prod, a_{12}=-q \beta S, a_{13}=-\delta S$, $a_{14}=-l \beta S+\delta S, a_{15}=\delta(\theta-1) S, a_{21}=\beta(I+q E+l J), a_{22}=q \beta S+\delta(I+J+(\theta-1) T)-\kappa-\Pi$, $a_{23}=\beta S+\delta E, a_{24}=l \beta S+\delta E, a_{25}=\delta(\theta-1) E, a_{32}=\kappa$, $a_{33}=-(\Pi+\alpha+\delta)+\delta I+\delta(I+J+(\theta-1) T), a_{34}=\delta I, a_{35}=\delta(\theta-1) I$, $a_{43}=\alpha+\delta J, a_{44}=-\left(\Pi+\gamma_{1}+\varsigma\right)+\delta J+\delta(I+J+(\theta-1) T), a_{45}=\delta(\theta-1) J$, $a_{53}=\delta T, a_{54}=\delta T+\varsigma, a_{55}=-\left(\Pi+\gamma_{2}\right)+\delta(\theta-1) T+\delta(I+J+(\theta-1) T+(\theta-1))$, $a_{63}=\delta R, a_{64}=\gamma_{1}+\delta R, a_{65}=\gamma_{2}+\delta(\theta-1) R, a_{66}=\delta(I+J+(\theta-1) T)-\Pi$, $a_{16}=a_{26}=a_{31}=a_{36}=a_{41}=a_{42}=a_{46}=a_{51}=a_{52}=a_{56}=a_{61}=a_{62}=0$
The Routh-Hurwitz Conditions with Diffusion are given as:

$C_{1}: q_{1}>0$

$C_{2}: q_{6}>0$

$C_{3}: \frac{q_{1} q_{2}-q_{3}}{q_{1}}>0$

$C_{4}: \frac{q_{1} q_{2} q_{3}-q_{3}^{2}-q_{1}^{2} q_{4}-q_{1} q_{5}}{q_{1} q_{2}-q_{3}}>0$

$C_{5}:=\frac{q_{3}^{2} q_{4}-q_{2} q_{3} q_{5}+q_{5}^{2}+q_{1}^{2}\left(q_{4}^{2}-q_{2} q_{6}\right)+q_{1}\left(q_{2}^{2} q_{5}-q_{2} q_{3} q_{4}-2 q_{4} q_{5}+q_{3} q_{6}\right)}{q_{3}^{2}+q_{1}^{2} q_{4}-q_{1}\left(q_{2} q_{3}+q_{5}\right)}>0$ $q_{3}^{2}+q_{1}^{2} q_{4}-q_{1}\left(q_{2} q_{3}+q_{5}\right)$

$C_{5}:=\frac{q_{3}^{2} q_{4} q_{5}-q_{2} q_{3} q_{5}^{2}+q_{5}^{3}-q_{3}^{3} q_{6}+q_{1}^{3} q_{6}^{2}+q_{1}^{2}\left(q_{4}^{2} q_{5}-q_{3} q_{4} q_{6}-2 q_{2} q_{5} q_{6}\right)+q_{1}\left(q_{2}^{2} q_{5}^{2}+q_{2} q_{3}\left(q_{3} q_{6}-q_{4} q_{5}\right)+q_{5}\left(3 q_{3} q_{6}-2 q_{4} q_{5}\right)\right)}{q_{3} q_{4}-q_{2} q_{5}+q_{5}+q_{1}\left(q_{4}^{2}-q_{2} q_{5}\right)+q_{1}\left(q_{2} q_{5}-q_{2} q_{5}-2 q_{5}+q_{5}\right)}>0$ $q_{3}^{2} q_{4}-q_{2} q_{3} q_{5}+q_{5}^{2}+q_{1}^{2}\left(q_{4}^{2}-q_{2} q_{6}\right)+q_{1}\left(q_{2}^{2} q_{5}-q_{2} q_{3} q_{4}-2 q_{1} q_{5}+q_{3} q_{5}\right)$ 